\title{
AMCoR
}

Asahikawa Medical College Repository http://amcor.asahikawa-med.ac.jp/

Nuclear Medicine Communications (2008.12) 29巻12号:1081 1085.

Cerebral control of bladder storage in patients with detrusor overactivity

Yafu Yin, Noriyuki Shuke、 Shigeo Kaneko, Atsutaka Okizaki, Junichi Sato、 Tamio Aburano、 Yaming Li、 Mitsuhiro Mizunaga、 Sunao Yachiku 


\section{Cerebral control of bladder storage in patients with detrusor}

\section{overactivity}

Yafu Yin ${ }^{1,2}$, Noriyuki Shuke ${ }^{1}$, Shigeo Kaneko ${ }^{3}$, Atsutaka Okizaki ${ }^{1}$, Junichi Sato ${ }^{1}$, Tamio Aburano ${ }^{1}$, Yaming $\mathrm{Li}^{2}$, Mitsuhiro Mizunaga ${ }^{3}$, Sunao Yachiku ${ }^{3}$

${ }^{1}$ Department of Radiology, Asahikawa Medical College, Asahikawa, Japan; ${ }^{2}$ Department of Nuclear Medicine, No.1 Hospital of China Medical University, China Medical University, Shenyang, China; ${ }^{3}$ Department of Urology, Asahikawa Medical College, Asahikawa, Japan

For correspondence or reprints contact:

Name: Yafu Yin, MD

Address: Department of Nuclear Medicine, No.1 Hospital of China Medical University, China Medical University, Nanjing North St. 155, Heping Ward, Shenyang 110001, China.

Telephone: +86-024-83282611

Fax: +86-024-22703576

E-mail: yinyafu@hotmail.com

Running title: Cerebral control of bladder storage

The words count: 2550 


\title{
Cerebral control of bladder storage in patients with detrusor overactivity
}

\begin{abstract}
Objective: To identify the brain areas which control the bladder storage by Tc-99m HMPAO SPECT with Neurological Statistical Image Analysis Software (NEUROSTAT) in patients with detrusor overactivity (DO).
\end{abstract}

Methods: SPECT scans were performed on nineteen patients with DO. And eight patients with normal detrusor function, who have some storage symptoms of lower urinary tract as the patients with DO, also had SPECT scan, as controls. All the subjects were male and right-handed, and were scanned twice under two conditions: resting state and urine withholding state. NEUROSTAT was adopted to analyze the difference in brain blood perfusion between groups and states. The results were displayed on Z-score images at a significance threshold of $P<0.05$ with correction for multiple comparisons.

Results: No region reached significant threshold in comparison of patients with DO and normal detrusor function in resting state. There were significant increases in tracer activity in the right inferior frontal gyrus and the right middle temporal gyrus during urine withholding 
state as compared with resting state in patients with normal detrusor function. Among the regions, the right inferior frontal gyrus was distinctly prominent at both Z-score and the extent. The regions that were activated significantly in patients with normal detrusor function, did not reach significant threshold during urine withholding in patients with DO.

Conclusions: The study indicated that the right inferior frontal gyrus and the right middle temporal gyrus, especially the right inferior frontal gyrus, played a role in cerebral control of bladder storage, inhibiting the contraction of detrusor in urine storage.

Key words: bladder storage; detrusor overactivity; NEUROSTAT; Tc-99m HMPAO; SPECT

\section{INTRODUCTION}

The principal functions of the urinary bladder are storage of urine, and evacuation of intravesical content by a coordinated series of neural and muscular events. The capacity for urine storage is assured by the distensibility of the epithelial, muscular, and connective tissue components of the urinary detrusor muscle (1). The disorder of urine storage would result in storage symptoms of lower urinary tract, such as urinary incontinence, increased daytime frequency and nocturia. 
Some lesion studies (2-5) and functional imaging studies in normal humans (6-8) have shown that the control of bladder storage was associated with some regions of the frontal cortex. The previous study has indicated that the bilateral inferior frontal gyri and the right superior and middle temporal gyri were activated during urine withholding as compared with resting state in healthy men (9). While little is known about the cerebral activation in patients with disorder of urine storage, which could further attest the cerebral control of bladder storage in detail. So, in this study, we compared the brain blood perfusion during urine withholding with that in resting state to identify the brain areas responsible for the control of bladder storage in the patients with some storage symptoms of lower urinary tract with detrusor overactivity (DO). DO, as defined by the International Continence Society (ICS) committee in 2002 (10), was an urodynamic observation characterized by involuntary detrusor contractions during the filling phase, which may be spontaneous or provoked. SPECT is a non-invasive and functional imaging technique used to study changes in the brain blood perfusion in humans. Neurological Statistical Image Analysis Software (NEUROSTAT) is a powerful technique for statistical analysis of the brain perfusion SPECT. Both of them were adopted as in the previous study (9). 


\section{MATERIALS AND METHODS}

\section{Subjects}

Nineteen patients (mean age $74.05 \pm 7.55$ years, range $59-83$ years), who were diagnosed as DO by urodynamic studies (UDS), were recruited. And the other eight patients (mean age $68.30 \pm 11.33$ years, range $48-77$ years), who were confirmed as normal detrusor function by UDS, were recruited as control. All the subjects had some storage symptoms of lower urinary tract, increased daytime frequency, nocturia, urgency or urinary incontinence, which were based on the definition of ICS (11). Increased daytime frequency is defined as "the complaint by the patient who considers that he/she voids too often by day". Nocturia is newly defined as "the complaint that the individual has to wake at night one or more times to void". The definition of Urgency is "the complaint of a sudden compelling desire to pass urine, which is difficult to defer". Urinary incontinence is defined as "the complaint of any involuntary leakage of urine”.

All of them underwent UDS, which included Uroflowmetry, Cystometry and Sphincter

electromyography, and CT or MRI of the brain. None of the patients had a spinal cord disorder or injury, peripheral innervation disorder, history of pelvic irradiation, current genitourinary tract infection, bladder occupying disease, or with detrusor sphincter dyssynergia (DSD).

The present study was approved by the Ethics Committee of Asahikawa Medical College. And written informed consent was given prior to entry for each subject, and all the subjects 
received full information as to the study plan.

\section{Data Acquisition}

Each subject was scanned twice without movement in a supine position. First, in resting state, 444MBq (12mCi) of Tc-99m HMPAO was injected intravenously while the subject lay supinely on the scanning table with empty bladder and eyes closed in a quiet examination room. Tc-99m HMPAO was generated by reconstituting HMPAO with freshly eluted Tc-99m pertechnetate and used within 30 minutes. Two minutes after the first injection, SPECT scan was performed for 10 minutes with a 4-head gamma camera (SPECT 2000H, Hitachi Medico., Tokyo, Japan), using low-energy high-resolution parallel-hole collimators. 64 projections were obtained on a $64 \times 64$ matrix in a step mode, with an acquisition time of 10 seconds per step and an energy window of $20 \%$ centered at $140 \mathrm{KeV}$. In-plane FWHM of this system was $7.4 \mathrm{~mm}$. Attenuation and scatter corrections were not performed.

After the first scan, 20mg furosemide was administered in a drop infusion, and additional 555MBq (15mCi) of Tc-99m HMPAO was injected intravenously when the subject sensed the urge to void in urine withholding state with full bladder. The condition of full bladder was confirmed by the subject's informing by moving his right index finger. The second scan was performed in the same way as the first. The subjects were permitted to void five minutes 
after the second injection. The interval between the first scan and the second one was between 15 and 37 minutes (mean 24 minutes). If any sensation or behavior changed in the subject, the examiner would record the changes.

Projection data were transferred to a dedicated data processing unit (Hitachi Gamma Vision RW-3000). The data were processed with a Butterworth filter (cutoff frequency: 0.55 cycles per centimeter; order: 8) and reconstructed into transaxial sections of 4.0-mm-thick images in planes parallel to the orbitomeatal line. Since the second scan data included the radioactivity from both resting and urine withholding states, the final image of urine withholding was obtained by subtracting the first scan data from the second. The image subtraction was performed after reconstruction by the data processing unit. The process was done with arithmetic operation that took the two scans as input and produced as output subtraction images whose pixel values were simply those of the second scan minus the corresponding pixel values from the first scan, thereby removing residual brain uptake of the first scan on the second scan.

\section{Statistical Analysis}

In this study, NEUROSTAT, which was developed by Minoshima et al. (12), was adopted to evaluate the change in brain blood perfusion. 
iSSP35_2tZ, analysis software of NEUROSTAT, which can conduct an unpaired $t$ test on two non-corresponding groups, was adopted to analyze the difference in the brain blood perfusion between Group I and Group II in resting state. It used the data output after analysis by iSSP35, which was also analysis software of NEUROSTAT. iSSP35 performed three-dimensional stereotactic surface projection (3D-SSP) analysis on the cross-sectional images of the subjects. It anatomically normalized the individual SPECT data to the standard brain and compared the regional pixel data with the normal SPECT database, then calculated the Z-score [(normal mean - individual value)/normal SD] for each pixel of the cerebral surface, and displayed the sites at which pixel value is statistically reduced or increased. After the analysis of iSSP35, the brain surface extraction data files were used to perform unpaired $t$ test and create Z-score image by iSSP35_2tZ. The results were shown on two Z-score images (increase and decrease images).

Stat_1tZ, analysis software of NEUROSTAT, which can conduct a paired $t$ test of two corresponding groups using cross-sectional images, was adopted to analyze the change in the brain blood perfusion between urine withholding and resting states. The process of analysis included six steps and was done as follows: anatomic standardization was performed firstly after co-registration of the two corresponding images, and then the pixel count was 
normalized. Averaging of subtraction image was done after voxel by voxel subtraction of the two corresponding images. The last steps were performing paired $t$ test and creating $Z$-score image in which $Z$-score was calculated by transforming the $t$ statistic to the normal distribution using a probability integral transformation. Results were shown on two Z-score images (increase and decrease images), and detailed information was recorded in a text file.

Since there were so many voxel-by-voxel comparisons, many $t$ values would reach a conventional level of significance by chance. Considering this, the correction for multiple comparisons is necessary. So the statistical significance was defined as $P<0.05$ with correction for multiple comparisons (13).

\section{RESULTS}

In the present study, fifty-four SPECT scans were obtained in total, and no changes including pain were recorded, except index finger moving. The results of CT and/or MRI and SPECT were in Table 1 and 2.

The results of iSSP35_2tZ with correction for multiple comparisons $(P<0.05)$

The statistical significance was defined as $P<0.05$ with correction for multiple comparisons, the corresponding $Z$ value was more than $4.530(P<0.000003)(14,15)$. 
No region with increased or decreased activity reached significant threshold in comparison of patients with DO and normal detrusor function in SPECT.

The results of Stat_1tZ with correction for multiple comparisons $(P<0.05)$

The statistical significance was defined as $P<0.05$ with correction for multiple comparisons, the corresponding $Z$ value was more than $4.35(P<0.000007)$ in patients with DO and $4.414(P<0.000005)$ in patients with normal detrusor function, respectively.

In patients with DO, only the left middle temporal gyrus (MTG) and middle frontal gyrus (MFG) were activated significantly with a cluster size of 20 and 3 voxels, respectively, during urine withholding state, as compared with resting (Table 3; Fig. 1).

In patients with normal detrusor functin, there were significant increases in tracer activity in bilateral inferior frontal gyrus (IFG) and the right MTG with a cluster size of 147, 20 and 58 voxels, respectively, during urine withholding state, as compared with resting, (Table 3; Fig. 2).

In both of the two groups, no region with decreased activity reached significant threshold in SPECT.

In this study, the voxel size is $4 \mathrm{~mm} \times 4 \mathrm{~mm} \times 4 \mathrm{~mm}$, and FWHM of SPECT was $7.4 \mathrm{~mm}$. So, in voxel-based comparisons with SPECT, a minimum cut-off was established at 50 voxels. 
We will not consider the regions with voxels less than 50 .

\section{DISCUSSION}

The aim of the present study was to identify the brain areas that control bladder storage by performing SPECT on the patients with DO and patients with normal detrusor function. According to the results of iSSP35_2tZ, no region reached significant threshold in comparison of the two groups in resting state. According to the results of Stat_1tZ, the right IFG and the right MTG were activated significantly during urine withholding, as compared with resting state in patients with normal detrusor function. Among these regions, the right IFG was the most prominent at both Z-score and the extent of the region. These regions were not activated significantly during urine withholding in patients with DO. The previous study identified that the right IFG and the right MTG were activated in healthy men, too (9). The results indicated that the right IFG and the right MTG were associated with the control of detrusor, which is one primary factor of bladder storage(1). As we know, during the urine storage phase, the detrusor is inhibited from contraction, and the striated sphincter is inhibited from relaxation. This negative feedback mechanism ensures intact continence and prevents the release of urine. Furthermore, it has been confirmed that the lesions above the 
pontine micturition center resulted in uninhibited bladder contractions (DO) attributed to a lack of inhibition from the cerebral cortex, while leaving relaxation of the urethral sphincter intact (16-18). So we could say that the right IFG and the right MTG, especially the right IFG, play a role in control of bladder storage, inhibiting the detrusor contraction, and the damage of which would result in DO.

In the present study, the left MTG and MFG were activated during urine withholding as compared with resting state in patients with DO, but the two gyri were not significant in fact for with only 20 and 3 voxels, respectively.

This is the first time that SPECT with NEUROSTAT was adopted to study the cerebral control of bladder storage in the patients with DO and patients with normal detrusor function. Although both Statistical Parametric Mapping (SPM) and NEUROSTAT are powerful techniques for statistical analysis of the brain perfusion SPECT, NEUROSTAT was found to be more suitable than SPM in anatomical standardization of atrophied brains (19). Thereby, NEUROSTAT is proper for the present study. However, both NEUROSTAT and SPM methods could produce artifacts(19), as also seen in clinical data. Although the number of voxels that are aligned consistently across subjects far exceeds that of mismatched voxels, such mismatches introduce a false functional reduction in atrophied brains. A need for 
further improvement of both algorithms is indicated.

Reviewing the brain imaging studies of bladder control in subject with normal control and

incontinence found that the bladder control depends on an extensive network of brain regions, dysfunction in various parts may contribute to incontinence, suggesting that there are different phenotypes requiring different treatment(20). The present study would be very useful for understanding the cerebral control of bladder storage and for the clinicians to identify the cause of bladder storage disorder and to decide the treatment, especially for the patients with DO. Of course, ROI analysis on more patients data would be necessary for proving the diagnostic value of this test in the individual patients with DO. And in the next phase, we will try to address this important issue.

One of the limitations of the present study was lack of healthy elder men with same ages as control. The other limitations, which included the brain activation due to urine withholding or/and some somatic discomfort, the effect of furosemide and the moving of the index finger has been discussed in the previous study (9).

\section{CONCLUSION}

In this SPECT study, no region with increased or decreased activity was found in 
comparison of the patients with DO and the patients with normal detrusor function in resting state. The right IFG and MTG were activated significantly during urine withholding in the patients with normal detrusor function, and were not found in the patients with DO. The present study indicated that the right IFG and MTG, especially the right IFG, played an important role in cerebral control of bladder storage, inhibiting the contraction of detrusor in urine storage, and the damage of which would result in DO.

\section{References}

1. Bradley W \& Scott F. Physiology of the urinary bladder. In: Harrison Jh Gr, Perlmutter Ad, Stamey Ta, Walsh Pc, ed. Campbell's UROLOGY. Philadelphia: W.B Saunders Company; 1978; p. 87-124.

2. Ueki K. Disturbances of micturition observed in some patients with brain tumor. Neurol Medico Chir.1960; 2: 25-33.

3. Cummings Jl. Vascular subcortical dementias: clinical aspects. Dementia.1994; 5: 177-180.

4. Maurice-Williams Rs. Micturition symptoms in frontal tumours. $J$ Neurol Neurosurg Psychiatry.1974; 37: 431-436.

5. Sakakibara R, Hattori T, Yasuda K \& Yamanishi T. Micturitional disturbance after acute hemispheric stroke: analysis of the lesion site by CT and MRI. J Neurol Sci.1996; 137: 47-56.

6. Blok Bf, Sturms Lm \& Holstege G. Brain activation during micturition in women. Brain.1998; 121 ( Pt 11): 2033-2042. 
7. Athwal Bs, Berkley Kj, Hussain I, et al. Brain responses to changes in bladder volume and urge to void in healthy men. Brain.2001; 124: 369-377.

8. Griffiths D, Derbyshire S, Stenger A \& Resnick N. Brain control of normal and overactive bladder. J Urol.2005; 174: 1862-1867.

9. Yin Y, Shuke N, Okizaki A, et al. Cerebral activation during withholding urine with full bladder in healthy men using 99mTc-HMPAO SPECT. $J$ Nucl Med.2006; 47: 1093-1098.

10. Abrams P, Cardozo L, Fall M, et al. The standardisation of terminology of lower urinary tract function: report from the Standardisation Sub-committee of the International Continence Society. Neurourol Urodyn.2002; 21: 167-178.

11. Abrams P, Cardozo L, Fall M, et al. The standardisation of terminology in lower urinary tract function: report from the standardisation sub-committee of the International Continence Society. Urology.2003; 61: 37-49.

12. Minoshima S, Koeppe Ra, Frey Ka \& Kuhl De. Anatomic standardization: linear scaling and nonlinear warping of functional brain images. J Nucl Med.1994; 35: 1528-1537.

13. Friston Kj, Frith Cd, Liddle Pf \& Frackowiak Rs. Comparing functional (PET) images: the assessment of significant change. J Cereb Blood Flow Metab.1991; 11: 690-699.

14. Minoshima S, Giordani B, Berent S, et al. Metabolic reduction in the posterior cingulate cortex in very early Alzheimer's disease. Ann Neurol.1997; 42: 85-94.

15. Worsley Kj. A unified statistical approach for determining significant signals in location and scale space images of cerebral activation. In: Myers R Cv, Baily D, Et Al., ed. Quantification of brain function using PET. San Diego: Academic; 1996; p. 327-333.

16. De Groat Wc. A neurologic basis for the overactive bladder. Urology.1997; 50: 36-52; discussion 53-36.

17. Fall M, Ohlsson Bl \& Carlsson Ca. The neurogenic overactive bladder. Classification based on 
urodynamics. Br J Urol.1989; 64: 368-373.

18. Hampel C, Wienhold D, Benken N, Eggersmann C \& Thuroff Jw. Definition of overactive bladder and epidemiology of urinary incontinence. Urology.1997; 50:4-14; discussion 15-17.

19. Ishii K, Willoch F, Minoshima S, et al. Statistical brain mapping of 18F-FDG PET in Alzheimer's disease: validation of anatomic standardization for atrophied brains. $J$ Nucl Med.2001; 42: 548-557.

20. Griffiths D \& Tadic Sd. Bladder control, urgency, and urge incontinence: Evidence from functional brain imaging. Neurourol Urodyn.2007. 
FIGURE 1. Results of Stat_1tZ in patients with DO. Increased activity in left MFG and MTG as arrows indicate.

FIGURE 2. Results of Stat_1tZ in patients with normal detrusor function. Increased activity in bilateral IFG and right MTG as arrows indicate. 

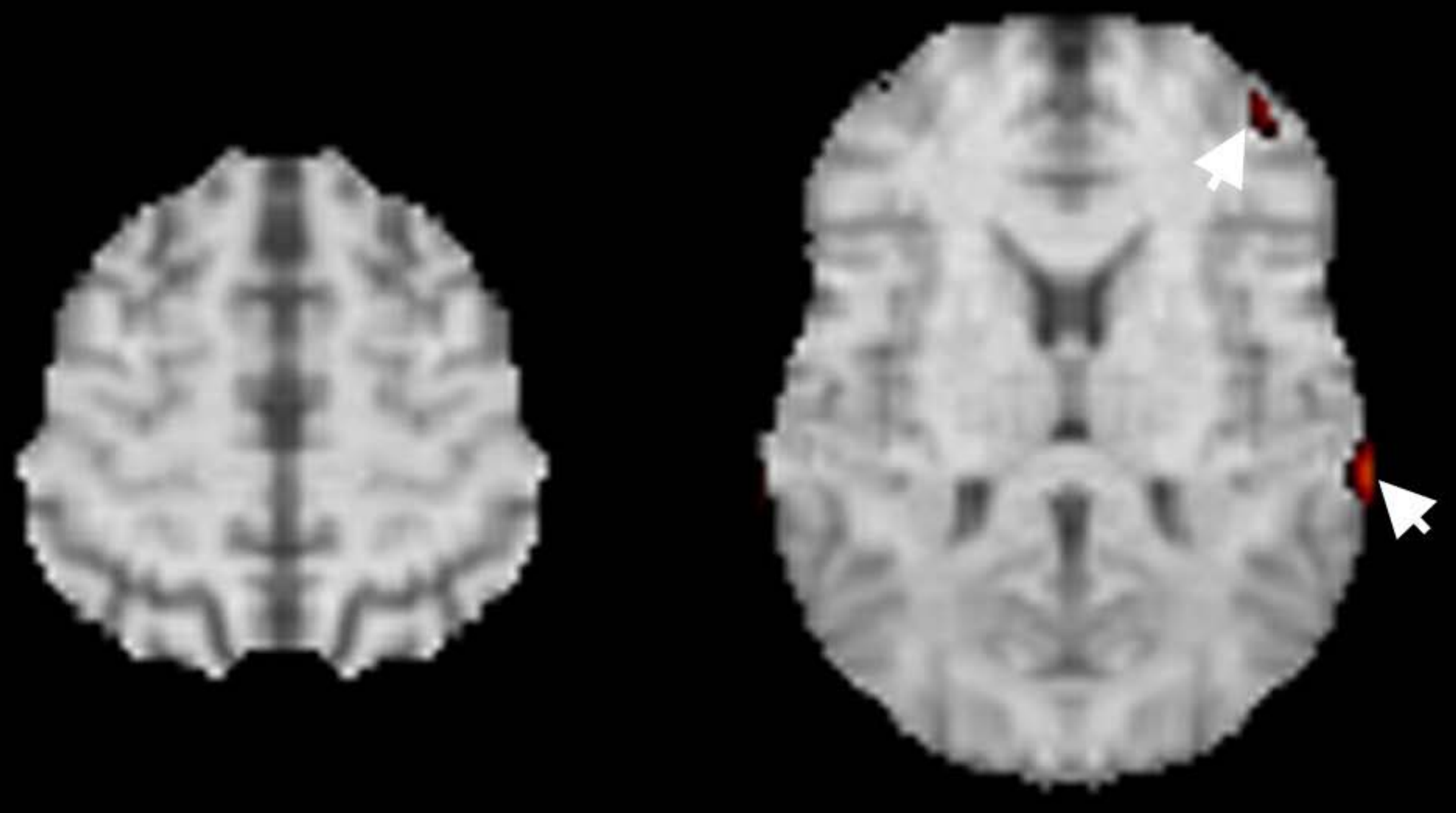

$Z$
6.23
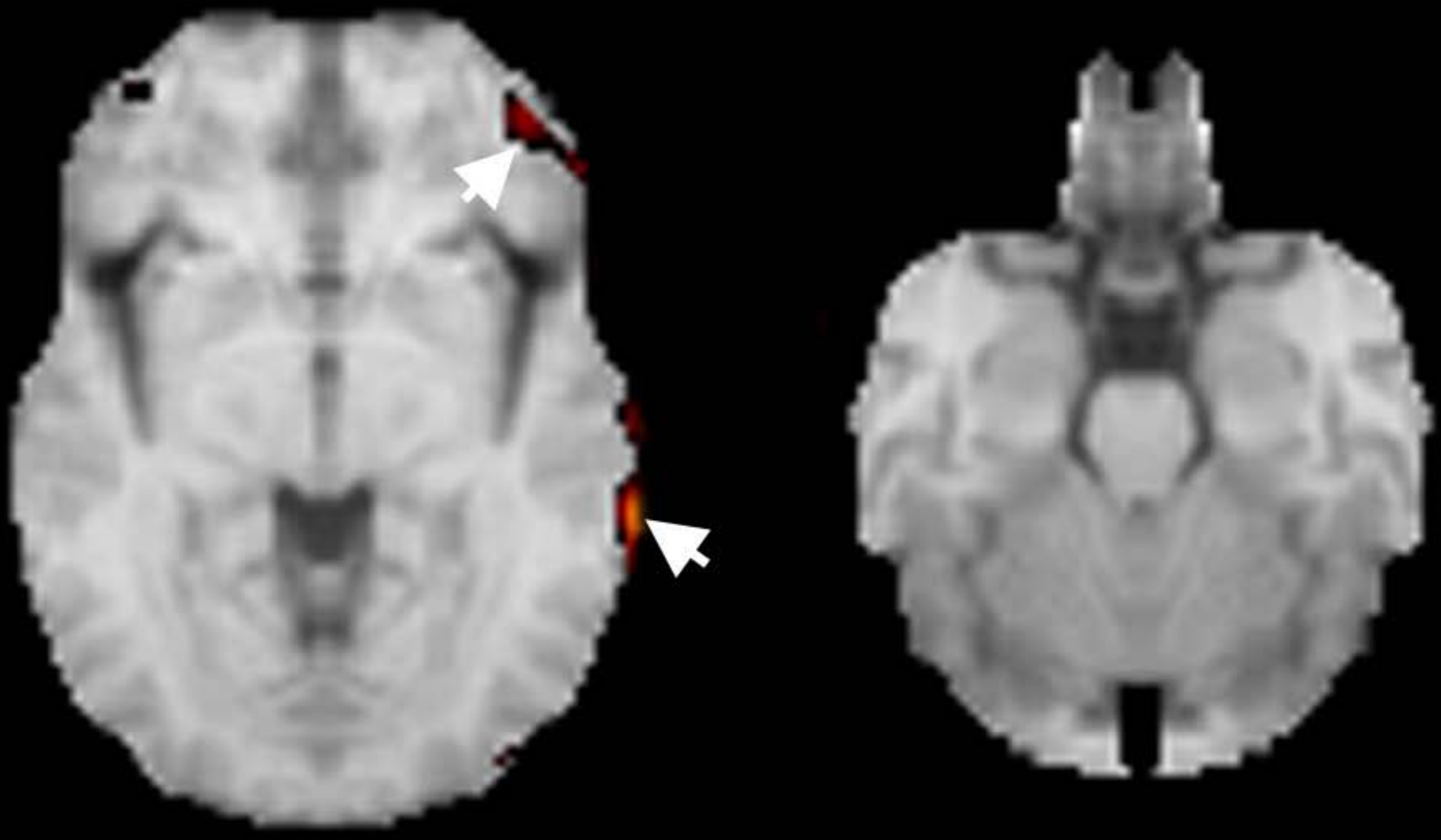

3.00 

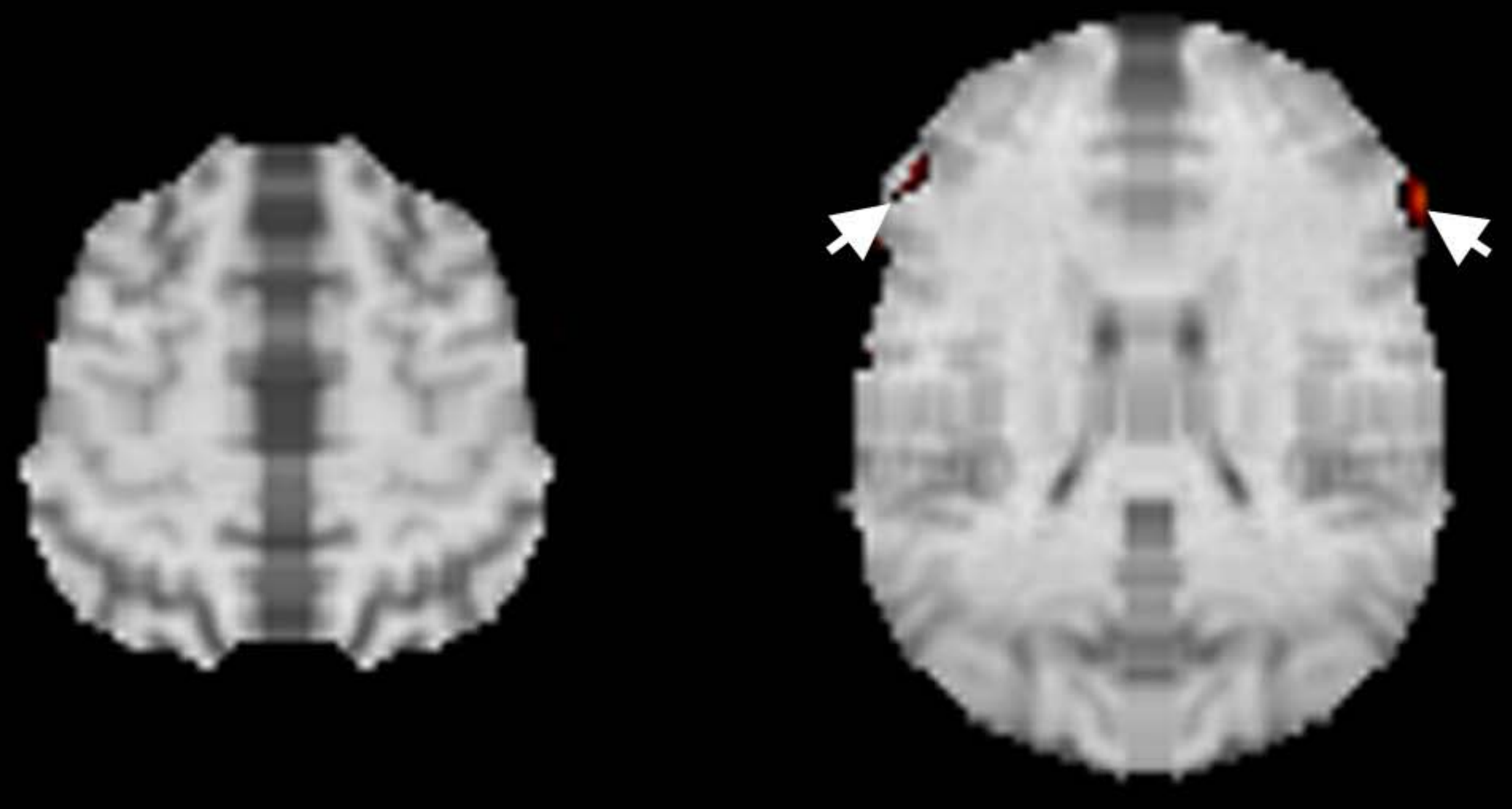

$Z$
6.23
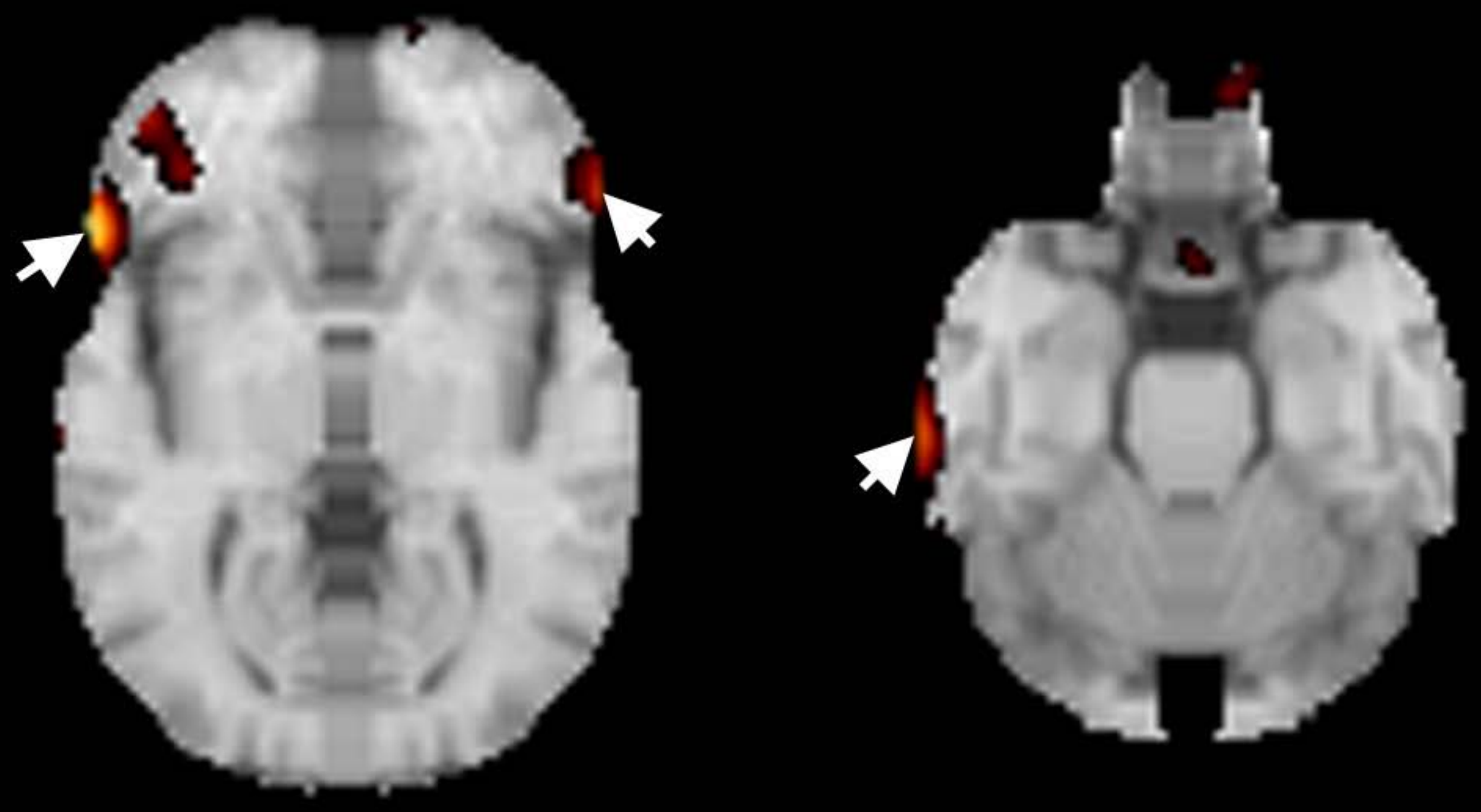

3.00 
Table 1 Results of CT and/or MRI

\begin{tabular}{lccc}
\hline & \multicolumn{3}{c}{ CT/MRI } \\
\cline { 2 - 4 } & Lacuna infarction & Lightly atrophied brain & Normal \\
\hline Group I (19) & 9 & 5 & 6 \\
Group II (8) & 2 & 2 & 5 \\
\hline
\end{tabular}


Table 2 Regions with decreased rCBF in SPECT in resting state

\begin{tabular}{lccccccccc}
\hline & \multicolumn{1}{c}{ SPECT } \\
\cline { 2 - 10 } & L. F. & R. F. & L. T. & R. T. & L. P. & R. P. & L./R. O. & R. Ce. & Normal \\
\hline Group I (19) & 6 & 5 & 4 & 3 & 1 & 1 & 0 & 1 & 10 \\
Group II (8) & 3 & 0 & 1 & 0 & 0 & 0 & 0 & 0 & 5 \\
\hline
\end{tabular}

L.: left; R.: right; F.: frontal lobe; T.: temporal lobe; P.: parietal lobe; O.:occipital lobe; Ce.: cerebrum 
TABLE 3. Results of Stat_1tZ

\begin{tabular}{|c|c|c|c|c|c|c|}
\hline \multirow[t]{2}{*}{ Groups } & \multirow{2}{*}{$\begin{array}{c}\text { Brain gyri with increased } \\
\text { activity* }\end{array}$} & \multicolumn{3}{|c|}{$\begin{array}{l}\text { Stereotactic coordinates of } \\
\text { peak activation }(\mathrm{mm}) * *\end{array}$} & \multirow[t]{2}{*}{$Z$-score $\dagger$} & \multirow{2}{*}{$\begin{array}{l}\text { Number of } \\
\text { voxels } !\end{array}$} \\
\hline & & $x$ & $y$ & $Z$ & & \\
\hline \multirow[t]{2}{*}{ Group I } & Left MTG (BA 21) & -73 & -37 & -4 & 4.73 & 20 \\
\hline & Left MFG (BA 10) & -48 & 57 & 4 & 4.46 & 3 \\
\hline \multirow[t]{3}{*}{ Group II } & Right IFG (BA45, 47) & 66 & 30 & -2 & 5.72 & 147 \\
\hline & Left IFG (BA47) & -62 & 35 & -7 & 4.81 & 20 \\
\hline & Right MTG (BA21) & 73 & -19 & -14 & 4.78 & 58 \\
\hline
\end{tabular}

*: Brain gyri with increased activity during urine withholding as compared with resting state.

**: Peak activations are indicated by $x, y$ and $z$ coordinates according to the stereotactic atlas of Talairach and Tournoux. Coordinates in standard stereotactic space: $x$ is distance to right $(+)$ or left (-) of the midsagittal line; $y$ is distance to anterior $(+)$ or posterior $(-)$ to vertical plane through the anterior commissure; $\mathrm{z}$ is the distance superior $(+)$ or inferior $(-)$ to the intercommissural (AC-PC) line. $\dagger$ : Z-score is the statistic value at the peak activation at a significance threshold of $P<0.05$ with correction for multiple comparisons. $t$ : The number of voxels means the total number of the voxels, which are continued at a significance threshold of $P<0.05$ with correction for multiple comparisons and include the corresponding peak voxel. 